\title{
Fontes de zinco aplicado em sementes de sorgo cv. BRS 310 e o crescimento inicial
}

\section{Zinc sources applied in seeds of sorghum $310 \mathrm{cv}$. BR and the initial growth}

\author{
Renato de Mello Prado ${ }^{1 *}$; Melissa de Castro Mouro ${ }^{2}$
}

\begin{abstract}
Resumo
A aplicação de zinco em sementes de sorgo poderá afetar a nutrição e o crescimento inicial das plantas. Assim, objetivou avaliar a aplicação de zinco na forma de sulfato e de óxido sobre a nutrição e o crescimento inicial da planta do sorgo cv. BRS 310. Os tratamentos foram constituídos de cinco doses 0 ; 14,$3 ; 28,6 ; 57,2 \mathrm{e} 114,4 \mathrm{~g} \mathrm{~kg}^{-1}$ de semente e de duas fontes de zinco, o sulfato ( $22 \%$ de $\mathrm{Zn}$ ) e o óxido ( $50 \%$ de $\mathrm{Zn}$ ). Cada unidade experimental constituída de bandeja de polietileno translúcido preenchida com $5 \mathrm{~L}$ de areia grossa lavada, com 50 sementes do sorgo cv. BRS 310. Aos 25 dias após a semeadura, efetuouse o corte das plantas, avaliou-se a massa seca da parte aérea e das raízes e determinou-se o teor e o acúmulo de Zn nas plantas. A aplicação de zinco na forma de óxido promoveu maior incremento de matéria seca em plantas de sorgo e proporcionou maior eficiência de utilização do micronutriente, embora, com menor eficiência de absorção, quando comparado a fonte sulfato. A fonte sulfato causou diminuição na produção da massa seca do sorgo. As fontes promoveram alteração na absorção de outros nutrientes pelas plantas de sorgo, sendo que a fonte sulfato incrementou a absorção de $\mathrm{P}, \mathrm{Ca}, \mathrm{S}$ e $\mathrm{Mn}$ e a fonte óxido maior absorção de $\mathrm{K}, \mathrm{Mg}$, $\mathrm{Cu}$ e Fe.
\end{abstract}

Palavras-chave: Sorghum bicolor, Zn, adubação, absorção

\begin{abstract}
The zinc application in sorghum seeds can affect the nutrition and the initial growth of the plants. Thus, the objective of their study was to evaluate the zinc application in the form of sulphate and oxide on the nutrition and on the initial growth of the plant of sorghum cv. BRS 310. The treatments had been five rates $0 ; 14,3 ; 28,6 ; 57,2$ and $114,4 \mathrm{~g} \mathrm{Zn} \mathrm{kg}^{-1}$ of seeds and two zinc sources, the sulphate (22\% of $\mathrm{Zn}$ ) and the oxide (50\% of $\mathrm{Zn})$. The experimental units were translucent polyethylene trays a filled with $5 \mathrm{~L}$ of washed thick sand, with 50 seeds of sorghum cv. BRS 310. The plants were cut 25 days after the sowing, and were evaluated for dry matter of above ground parts and the roots and it was determined the content and the accumulation of $\mathrm{Zn}$ in the plants. The zinc application in the oxide form promoted greater increment of dry matter in sorghum plants and provided to greater efficiency of use of the micronutrient, even so, with lesser efficiency of absorption, when compared the source sulphate. The source sulphate caused reduction in the production of the mass of the dry matter of sorghum. The sources had promoted alteration in the absorption of other nutrients for the sorghum plants, being that the source sulphate developed the absorption of $\mathrm{P}, \mathrm{Ca}, \mathrm{S}$ and $\mathrm{Mn}$ and the source oxide bigger absorption of $\mathrm{K}, \mathrm{Mg}, \mathrm{Cu}$ and $\mathrm{Fe}$.
\end{abstract}

Key words: Sorghum bicolor, Zn, fertilization, absorption

1 Prof. Dr. Depto. de Solos e Adubos, Faculdade de Ciências Agrárias e Veterinárias, Unesp. E:mail: rmprado@ fcav.unesp.br

2 Graduanda em Zootecnia, Depto. de Solos e Adubos, Faculdade de Ciências Agrárias e Veterinárias, Unesp.

* Autor para correspondência

Recebido para publicação 28/09/06 Aprovado em 14/06/07 


\section{Introdução}

A deficiência de zinco em solos tropicais é generalizada, afetando vasta área produtora de grãos do Brasil, sobretudo nas áreas sob vegetação original de cerrado (LOPES, 1983). E associado a isso, práticas agrícolas inadequadas tais como excesso de calagem, pode agravar as desordens nutricionais nas culturas comprometendo a produção. Este fato, torna-se importante, devido a importância do zinco na nutrição das plantas, sendo a mais conhecida a sua participação na biossíntese do AIA (MARSCHNER, 1995), e atuando como ativador de uma série de enzimas importantes para o metabolismo das plantas. Neste sentido, diversos autores tem indicado resposta positiva de plantas de sorgo à aplicação de Zn (LOCKMAN, 1972; ALVAREZ; DEFELIPO; BARROS, 1978; RITCHEY et al., 1986).

Ao ser exigido em quantidades relativamente pequenas pelas plantas, a aplicação de zinco em sementes de sorgo pode ser uma forma promissora de adubação com o micronutriente. Na literatura, alguns trabalhos têm indicado que a aplicação de $\mathrm{Zn}$ em sementes é promissora, especialmente em cereais milho e o arroz (RIBEIRO; SANTOS; MENEZES, 1994; RIBEIRO e SANTOS, 1996). No sorgo (cv. BR304), Yagi et al. (2006) observaram que a aplicação de zinco em sementes, na forma de sulfato, não afetou o acúmulo da massa seca da parte aérea, entretanto, diminuiu o acúmulo da massa seca das raízes e da planta inteira. Estes resultados são contraditórios, tendo em vista que nos trabalhos citados anteriormente, são distintos as espécies, além das doses e fontes do nutriente. Assim, os benefícios da aplicação de zinco em sementes no crescimento inicial das plantas dependerá da dose adequada do nutriente e da fonte para garantir a adequada nutrição, sem riscos de fitoxicidade às plântulas.

Diante deste contexto, o presente trabalho objetivou avaliar a aplicação de zinco na forma de sulfato e óxido, sobre a nutrição e o crescimento inicial da cultura do sorgo cv. BRS 310 .

\section{Material e Métodos}

O experimento foi realizado em condições de casa de vegetação, da FCAV/Unesp, em Jaboticabal-SP. O delineamento experimental adotado foi o inteiramente casualizado, sendo testados cinco doses e duas fontes de zinco, com três repetições. Assim, os tratamentos foram cinco doses 0,$0 ; 14,3 ; 28,6 ; 57,2$ e $114,4 \mathrm{~g} \mathrm{~kg}^{-1}$ de semente e duas fontes de zinco, o sulfato ( $22 \%$ de $\mathrm{Zn}$ ) e o óxido ( $50 \%$ de $\mathrm{Zn}$ ).

A unidade experimental constituiu-se de uma bandeja (30 $\mathrm{cm}$ de comprimento, $20 \mathrm{~cm}$ de largura e $10 \mathrm{~cm}$ de altura) de polietileno translúcido preenchida com $5 \mathrm{~L}$ de areia grossa lavada, com 50 sementes do sorgo cv. BRS 310 .

A aplicação dos fertilizantes às sementes, procedeu-se inicialmente dissolução da respectiva fonte em um recipiente com $10 \mathrm{~mL}$ de água, adicionando-se a seguir, esta mistura, às sementes. Logo, e em seguida, efetuou-se a semeadura do sorgo nas bandejas contendo areia lavada. Considerou-se, assim, que a dose de $\mathrm{Zn}$ foi integralmente aplicada nas unidades experimentais (bandejas).

A irrigação foi realizada com água deionizada, por meio de reposição periódica do volume referente a $10 \%$ do peso da areia seca de cada recipiente. Aos 8 e 17 dias após a semeadura (DAS), em cada recipiente, foram aplicados a solução nutritiva completa (HOAGLAND; ARNON, 1950), com omissão do Zn.

Aos 25 DAS, efetuou-se o corte das plantas, separando-se a parte aérea e as raízes, que foram lavadas e secas em estufa de circulação forçada de $\operatorname{ar}\left(70^{\circ} \mathrm{C}\right)$, até atingirem massa constante. Nos tecidos vegetais, foi determinado o teor de macro e micronutrientes, conforme metodologia de Bataglia et al. (1983).

Com base nos resultados da massa da matéria seca da parte aérea e das raízes, e no teor de Zn, calculou-se o acúmulo do nutriente nas plantas. Além disso, obteve-se a eficiência de absorção (em $\mathrm{mg} \mathrm{g}^{-1}$ ) 
(acúmulo de $\mathrm{Zn}$ na planta/massa seca de raízes), a eficiência de transporte (em \%) (acúmulo de Zn na planta/acúmulo de $\mathrm{Zn}$ nas raízes x 100) e a eficiência de utilização (em $\mathrm{g} \mathrm{mg}^{-1}$ ) (massa seca total) ${ }^{2 /}$ (conteúdo total do nutriente na planta) (SIDDIQI; GLASS, 1981). Os dados foram submetidos análise de variância pelo teste $\mathrm{F}$, tendo as fontes comparadas pelo teste Tukey (5\% de probabilidade) e as doses analisadas pelo emprego de regressão polinomial. As análises foram processadas por meio do Statistical Analysis System (SAS INSTITUTE, 1996).

\section{Resultados e Discussão}

a) Efeito dos tratamentos na produção da massa de matéria seca e no teor e acúmulo de zinco

Observou-se efeito significativo dos fatores doses, fontes de zinco e da interação sobre a produção da massa seca (parte aérea, raízes e planta inteira), exceto para o efeito das doses na produção de massa seca das raízes; teor e acúmulo de zinco na parte aérea e nas raízes do sorgo (Tabela 1).

Tabela 1. Resumo da análise de variância referente à produção da massa seca, teor e acúmulo de zinco na parte aérea e raízes, em função da aplicação de diferentes fontes e doses de zinco às sementes de sorgo cv. BRS 310

\begin{tabular}{|c|c|c|c|c|c|c|c|c|}
\hline \multirow[t]{3}{*}{ Causa de variação } & \multicolumn{3}{|c|}{ Massa da matéria seca } & \multicolumn{2}{|c|}{ Teor de $\mathrm{Zn}$} & \multicolumn{3}{|c|}{ Acúmulo de $\mathrm{Zn}$} \\
\hline & \multicolumn{3}{|c|}{ 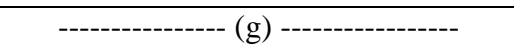 } & \multicolumn{2}{|c|}{----- $\left(\mathrm{mg} \mathrm{kg}^{-1}\right)$------ } & \multicolumn{3}{|c|}{------- $\left(\mathrm{mg} \mathrm{planta}^{-1}\right)$------ } \\
\hline & Parte aérea & Raiz & Planta inteira & Parte aérea & Raiz & Parte aérea & Raiz & $\begin{array}{l}\text { Planta } \\
\text { inteira }\end{array}$ \\
\hline \multicolumn{9}{|l|}{ Doses (D) } \\
\hline \multicolumn{9}{|l|}{$\mathrm{g} \mathrm{kg}^{-1}$} \\
\hline 0 & 1,6 & 0,7 & 2,3 & 25,0 & 50,0 & 0,04 & 0,03 & 0,07 \\
\hline 14,3 & 1,2 & 0,6 & 1,8 & 652,5 & 912,5 & 0,8 & 0,6 & 1,4 \\
\hline 28,6 & 1,2 & 0,6 & 1,9 & 735,0 & 841,7 & 0,9 & 0,5 & 1,5 \\
\hline 57,2 & 1,3 & 0,6 & 1,8 & 1393,5 & 1000,0 & 1,3 & 0,4 & 1,7 \\
\hline 114,4 & 1,4 & 0,6 & 2,0 & 1177,5 & 1075,0 & 1,5 & 0,6 & 2,1 \\
\hline Teste F & $20,53^{* * *}$ & $1,96^{\mathrm{ns}}$ & $13,87^{* * 2}$ & $180,75^{* * *}$ & $87,87^{* * *}$ & $149,90^{* *}$ & $54,88^{* *}$ & $165,06^{* * *}$ \\
\hline \multicolumn{9}{|l|}{ Fontes (F) } \\
\hline Sulfato 1 & $1,3^{\mathrm{b}}$ & $0,6^{\mathrm{b}}$ & $1,9^{\mathrm{b}}$ & $1237,0^{\mathrm{a}}$ & $970,0^{\mathrm{a}}$ & $1,4^{\mathrm{a}}$ & $0,5^{\mathrm{a}}$ & $1,9^{\mathrm{a}}$ \\
\hline Óxido 2 & $1,4^{\mathrm{a}}$ & $0,7^{\mathrm{a}}$ & $2,0^{\mathrm{a}}$ & $356,3^{\mathrm{b}}$ & $581,7^{\mathrm{b}}$ & $0,5^{\mathrm{b}}$ & $0,4^{\mathrm{b}}$ & $0,8^{\mathrm{b}}$ \\
\hline Teste F & $5,58^{*}$ & $14,25^{* *}$ & $16,66^{*}$ & $625,22^{* * *}$ & $96,08^{* *}$ & $521,34^{* * *}$ & $7,65^{*}$ & $382,64^{* * *}$ \\
\hline \multicolumn{9}{|l|}{ Interação } \\
\hline $\mathrm{D} \times \mathrm{F}$ & $64,13^{* *}$ & $20,89^{* * *}$ & $75,32^{* *}$ & $133,02^{* *}$ & $21,93^{* *}$ & $52,24^{* *}$ & $7,39^{* *}$ & $30,10^{* *}$ \\
\hline \multicolumn{9}{|l|}{ Desdobramentos } \\
\hline Fonte dentro D1 & $0,00^{\mathrm{ns}}$ & $0,00^{\mathrm{ns}}$ & $0,00^{\mathrm{ns}}$ & $0,00^{\mathrm{ns}}$ & $0,00^{\mathrm{ns}}$ & $0,00^{\mathrm{ns}}$ & $0,00^{\mathrm{ns}}$ & $0,00^{\mathrm{ns}}$ \\
\hline Fonte dentro D2 & $52,20^{* *}$ & $11,18^{* *}$ & $54,40^{* *}$ & $21,47^{* *}$ & $0,71^{\mathrm{ns}}$ & $75,45^{* *}$ & $0,94^{\mathrm{ns}}$ & $61,74^{* *}$ \\
\hline Fonte dentro D3 & $28,87^{* *}$ & $1,12^{\mathrm{ns}}$ & $21,13^{* *}$ & $20,89^{* *}$ & $34,01^{\text {** }}$ & $65,61^{* *}$ & $35,16^{* *}$ & $89,55^{* *}$ \\
\hline Fonte dentro D4 & $148,65^{* *}$ & $58,44^{* *}$ & $187,52^{* *}$ & $720,07^{* *}$ & $103,22^{* * *}$ & $236,96^{* *}$ & $0,09^{\mathrm{ns}}$ & $144,63^{* *}$ \\
\hline Fonte dentro D5 & $32,37^{* *}$ & $27,06^{* * *}$ & $54,89^{* * *}$ & $394,88^{* *}$ & $45,87^{* *}$ & $356,29^{* *}$ & $1,04^{\mathrm{ns}}$ & $207,14^{* *}$ \\
\hline Dose dentro F1 & $35,87^{* *}$ & $16,90^{* *}$ & $46,49^{* *}$ & $300,55^{* *}$ & $80,71^{* *}$ & $189,0^{* *}$ & $35,76^{* *}$ & $167,34^{* *}$ \\
\hline Dose dentro F2 & $48,78^{* * *}$ & $5,95^{* *}$ & $42,70^{* *}$ & $13,22^{* *}$ & $29,10^{* *}$ & $14,16^{* *}$ & $26,52^{* *}$ & $27,82^{* * *}$ \\
\hline $\mathrm{CV}(\%)$ & 6,7 & 15,60 & 6,3 & 12,1 & 14,0 & 12,1 & 17,9 & 10,7 \\
\hline
\end{tabular}

**; *; ns: resultados significativo pelo teste $\mathrm{F}$ a $1 \%$ e $5 \%$ e não significativo a $5 \%$, respectivamente. Letras iguais não diferem entre si pelo teste Tukey a $5 \%$ de probabilidade

Uma vez que a interação entre doses e fontes sobre produção de massa seca, realizou-se estudo de regressão para avaliar o efeito das fontes dentro do fator dose de zinco. Pelos resultados, o emprego da fonte óxido de zinco, promoveu aumento de forma quadrática na produção da massa seca da parte aérea, e linear para raízes e para planta inteira (Figuras 1 a,b,c). Entretanto, apresentou coeficiente de determinação baixo, foi pouco expressivo, embora significativo. Por sua vez, a aplicação da fonte sulfato 
de zinco, provocou efeito quadrático decrescente na massa seca das plantas (raiz e planta inteira) e linear para a parte aérea (Figuras 1 a,b,c). Esses resultados discordam do Ribeiro e Santos (1996) que relataram o maior ganho da massa seca com o emprego da fonte sulfato de zinco no tratamento de sementes para culturas como milho e arroz, em experimentos conduzidos em casa de vegetação.
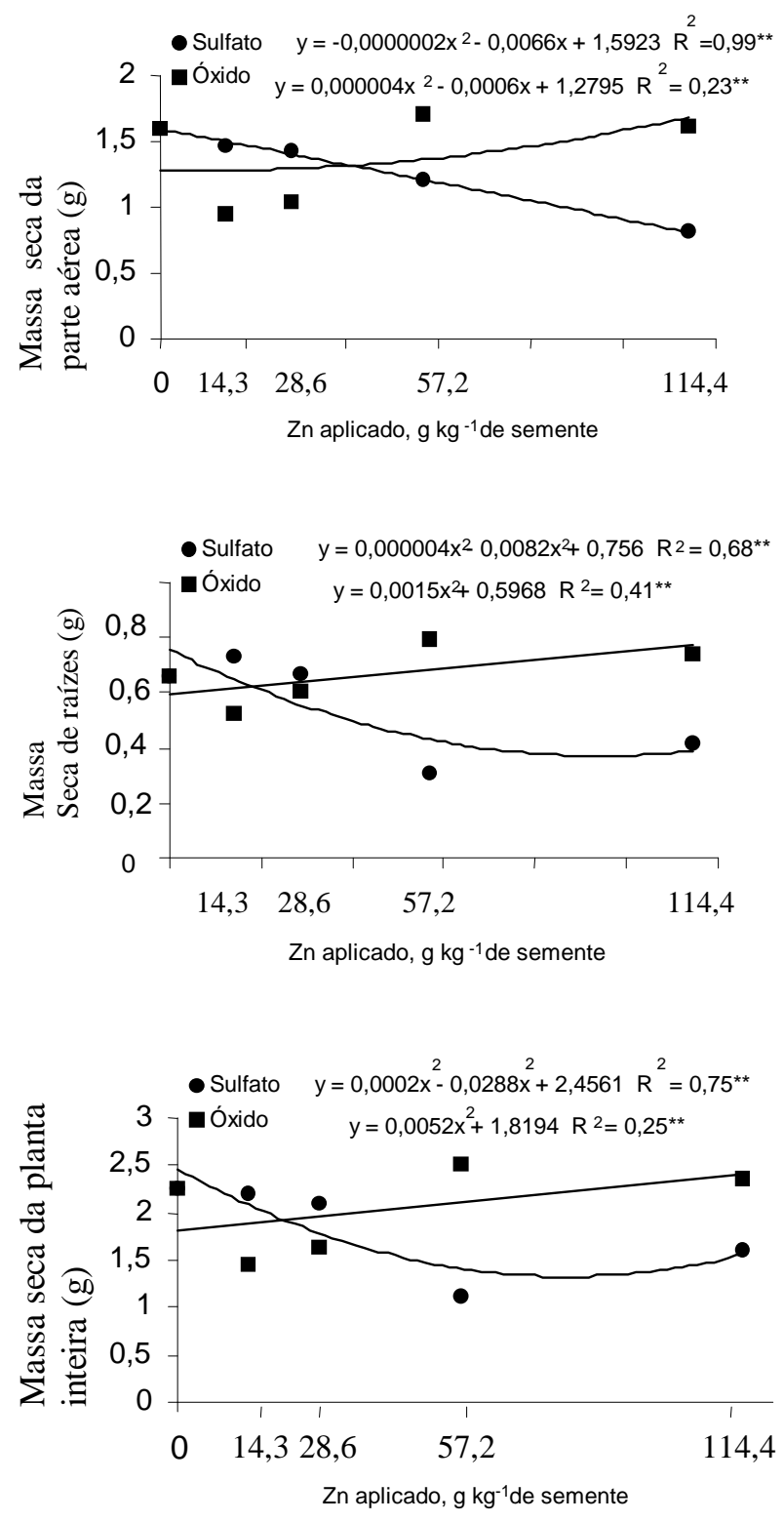

Figura 1. Efeito da aplicação de fontes de zinco em sementes de sorgo cv. BRS 310 na produção de massa seca da parte aérea (a), raízes (b) e total (planta inteira) (c) aos 25 dias após a semeadura.
A menor produção da massa seca da plantas com o uso da fonte sulfato, especialmente nas maiores doses de Zn (Figura 1), provavelmente deveu-se ao fato do excesso de zinco, que poderia ter causado inibição do elongamento radicular, que é um parâmetro característico de toxicidade (MARSCHNER, 1995), uma vez que foi observado que o aumento das doses de $\mathrm{Zn}$ propiciou elevação de forma quadrática do teor do nutriente tanto na parte aérea quanto nas raízes (Figura 2 a,b). Houve, também, acúmulo quadrático de Zn na parte aérea (Figura 2c) e nas raízes (Figura 2d) para as duas fontes. A aplicação de $\mathrm{Zn}$ na forma de sulfato na dose de 80 e $78 \mathrm{~g} \mathrm{~kg}^{-1}$, proporcionou o teor de $\mathrm{Zn}$ de 2.392 e $1.665 \mathrm{mg} \mathrm{kg}^{-1}$ na parte aérea e raízes, respectivamente (Figuras 2a,b). Ao passo que o uso do zinco na forma de óxido, os teores máximo de $\mathrm{Zn}$ foram bem menores, sendo que o conteúdo de $\mathrm{Zn}$ da parte aérea foi de $523 \mathrm{mg}$ $\mathrm{kg}^{-1}$ e nas raízes foi de $788 \mathrm{mg} \mathrm{kg}^{-1}$, com uso das doses 70 e $80 \mathrm{~g} \mathrm{~kg}^{-1}$, respectivamente (Figuras 2a,b). Portanto, observou-se uma maior absorção de $\mathrm{Zn}$ pelas plantas de sorgo submetidas ao uso do sulfato comparado ao óxido. Esse fato pode estar relacionado com a solubilidade das fontes, pois o $\mathrm{Zn}$ presente no sulfato é mais solúvel e disponível às plantas quando comparado a fonte óxido (VALE, 2001).

O uso da fonte óxido, na maior dose do nutriente, proporcionou teor de $\mathrm{Zn}$ na parte aérea de $364 \mathrm{mg}$ $\mathrm{kg}^{-1}$ (Figura 2a), e esteve associado com a maior produção de massa seca. Este resultado foi muito maior ao indicado por Cantarella et al. (1997) (15 e $50 \mathrm{mg} \mathrm{Zn} \mathrm{kg}^{-1}$ ) para a cultura do sorgo. Entretanto, essa indicação seria para plantas cultivadas em condições de campo e em folhas diagnósticas, diferentemente do presente trabalho, em que os resultados foram obtidos a partir de tecido da parte aérea em plantas em estádio inicial de crescimento. Por outro lado, Yagi et al. (2006) em um estudo com sorgo submetido a aplicação de $\mathrm{Zn}$ nas sementes, também observaram elevados teores de $\mathrm{Zn}$ na parte aérea (234 $\left.\mathrm{mg} \mathrm{kg}^{-1}\right)$, sem que tenha sido observado qualquer sintomas de toxicidade nas plantas. 

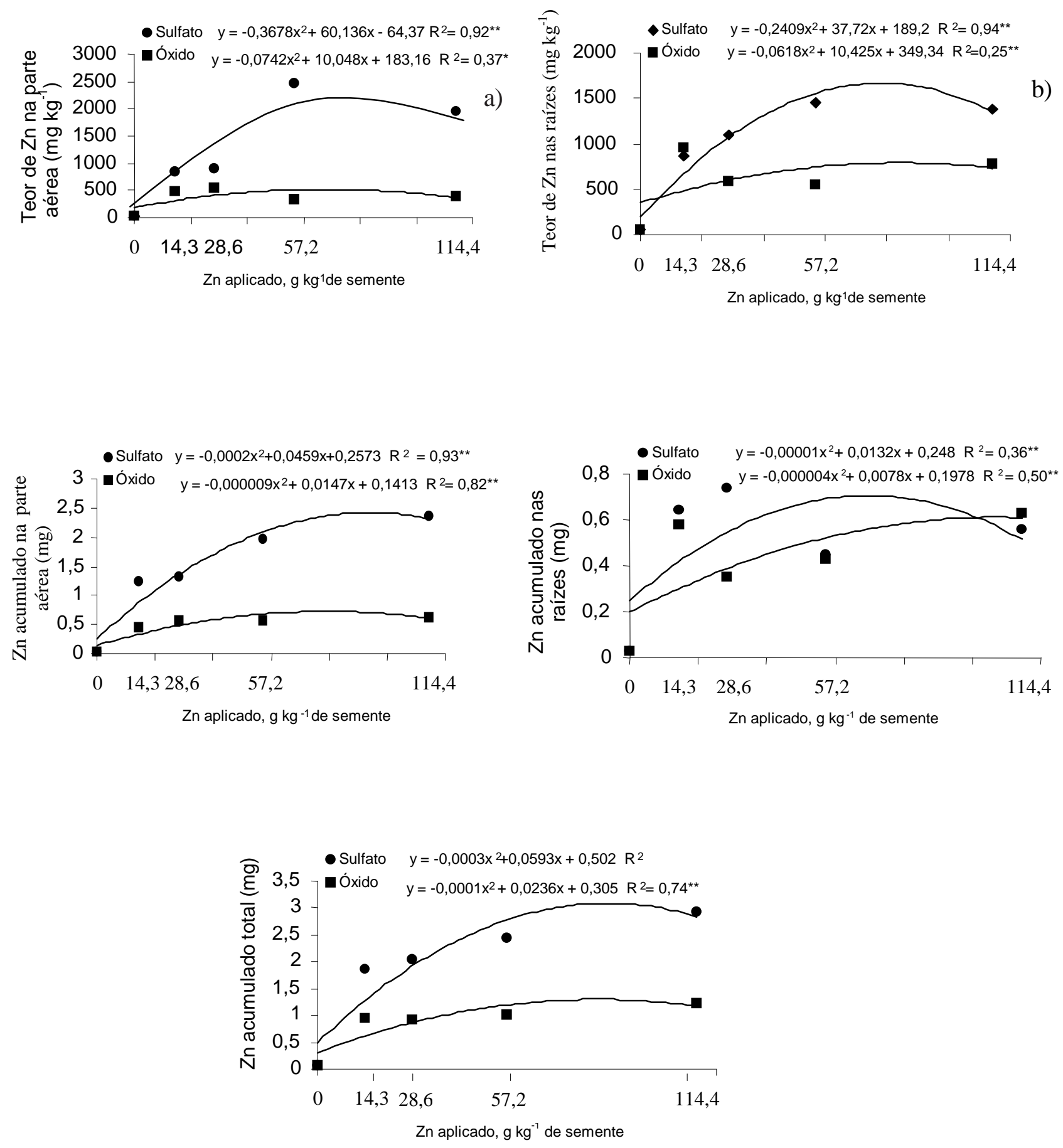

Figura 2. Efeito da aplicação de fontes de zinco em sementes de sorgo cv. BRS 310 no teor de Zn da parte aérea (a), raiz (b) e acúmulo de Zn da parte aérea (c), raízes (d) e total (planta inteira) (e) aos 25 dias após a semeadura. 
Tabela 2. Resumo da análise de variância referente à eficiência de absorção, transporte e de utilização do zinco, em função da aplicação de diferentes fontes e doses de Zn às sementes de sorgo cv. BRS 310

\begin{tabular}{|c|c|c|c|}
\hline Causa de variação & $\begin{array}{l}\text { Eficiência de } \\
\text { absorção }\end{array}$ & $\begin{array}{l}\text { Eficiência de } \\
\text { transporte }\end{array}$ & $\begin{array}{l}\text { Eficiência de } \\
\text { utilização }\end{array}$ \\
\hline \multicolumn{4}{|l|}{ Doses (D) } \\
\hline \multicolumn{4}{|l|}{$\mathrm{g} \mathrm{kg}^{-1}$} \\
\hline 0 & 0,1 & 54,9 & 70,3 \\
\hline 14,3 & 2,2 & 56,3 & 2,5 \\
\hline 28,6 & 2,3 & 63,1 & 2,5 \\
\hline 57,2 & 4,5 & 69,1 & 2,7 \\
\hline 114,4 & 4,5 & 66,7 & 3,4 \\
\hline Teste F & $83,99^{* *}$ & $7,48^{* * *}$ & $946,03^{* * *}$ \\
\hline \multicolumn{4}{|l|}{ Fontes (F) } \\
\hline Sulfato 1 & $4,2^{\mathrm{a}}$ & $69,3^{\mathrm{a}}$ & $15,3^{\mathrm{b}}$ \\
\hline Óxido 2 & $1,3^{\mathrm{b}}$ & $54,7^{\mathrm{b}}$ & $17,3^{\mathrm{a}}$ \\
\hline Teste $\mathrm{F}$ & $255,31^{* * *}$ & $51,09^{* *}$ & $4,97^{* *}$ \\
\hline \multicolumn{4}{|l|}{ Interação } \\
\hline $\mathrm{DXF}$ & $54,19^{* *}$ & $7,87^{* *}$ & $1,77^{\mathrm{ns}}$ \\
\hline \multicolumn{4}{|l|}{ Desdobramentos } \\
\hline Fonte dentro D1 & $0,00^{\mathrm{ns}}$ & $0,00^{\mathrm{ns}}$ & ---- \\
\hline Fonte dentro D2 & $3,44^{\text {ns }}$ & $16,94^{* *}$ & ---- \\
\hline Fonte dentro D3 & $14,61^{* *}$ & $0,20^{\mathrm{ns}}$ & ---- \\
\hline Fonte dentro D4 & $260,90^{* *}$ & $28,49^{* *}$ & ---- \\
\hline Fonte dentro D5 & $193,12^{* *}$ & $36,96^{* *}$ & --- \\
\hline Dose dentro F1 & $132,45^{* *}$ & $12,43^{* *}$ & --- \\
\hline Dose dentro F2 & $5,73^{* *}$ & $2,92^{*}$ & ---- \\
\hline $\mathrm{CV}(\%)$ & 18,1 & 9,0 & 14,7 \\
\hline
\end{tabular}

**; *; ns: resultados significativo pelo teste $\mathrm{F}$ a $1 \%$ e $5 \%$ e não significativo a 5\%, respectivamente. Letras iguais não diferem entre si pelo teste Tukey a $5 \%$ de probabilidade

As elevadas doses de $\mathrm{Zn}$ na forma de sulfato proporcionaram maior teor de $\mathrm{Zn}$ na parte aérea das plantas de sorgo (2.392 $\mathrm{mg} \mathrm{kg}^{-1}$ ) causaram fitotoxicidade. Neste caso, foram observados sintomas característicos de toxidez de $\mathrm{Zn}$, como diminuição do tamanho da planta e da área foliar, seguida de clorose, evoluindo para planta inteira, um pigmento pardo-avermelhado. A diminuição no tamanho das plantas pode estar associada ao fato de que no xilema das plantas intoxicadas acumularem tampões "plugs" contendo $\mathrm{Zn}$, os quais dificultam a ascensão da seiva bruta, além de interferir no metabolismo de carboidratos, inibindo o transporte de assimilados (MALAVOLTA et al., 1997). Em elevadas concentrações de Zn na planta, com desenvolvimento de sintomas de toxicidade podem afetar a produção da massa seca, e são amplamente relatados na literatura (MENGEL; KIRKBY, 1987). b) Efeito dos tratamentos sobre a eficiência nutricional de zinco

$\mathrm{O}$ efeito das diferentes doses e fontes de zinco, sobre a eficiências de absorção, transporte e utilização do Zn pelas plantas de sorgo, estão apresentados na Tabela 2.

A eficiência de absorção de Zn pelo sorgo aumentou com as doses de $\mathrm{Zn}$, para ambas fontes estudadas (Figura 3a). Com relação às fontes, foi observado que a eficiência de absorção foi maior quando se utilizou a fonte sulfato, comparada à fonte óxido, atingindo ponto de máximo de 8,3 e 1,77 mg de $\mathrm{Zn}$ acumulado $\mathrm{g}^{-1}$ da massa seca de raízes, respectivamente (Figura 3a). Assim, a eficiência de absorção de Zn com uso do sulfato foi superior em mais de quatro vezes maior à fonte óxido. 
a)

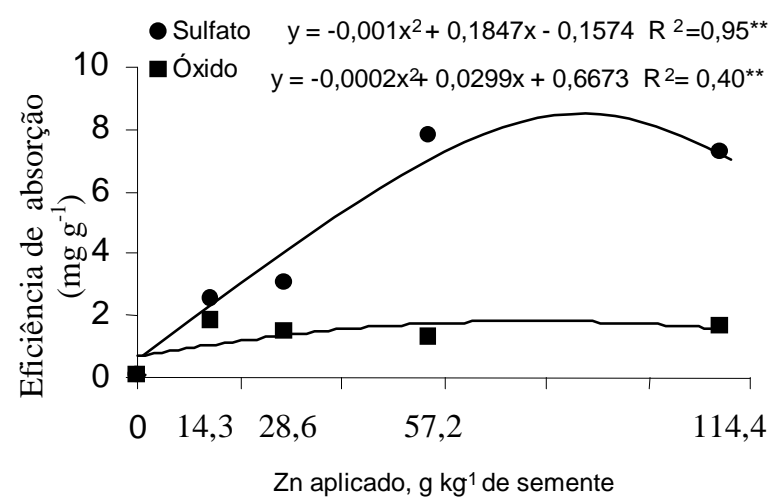

b)

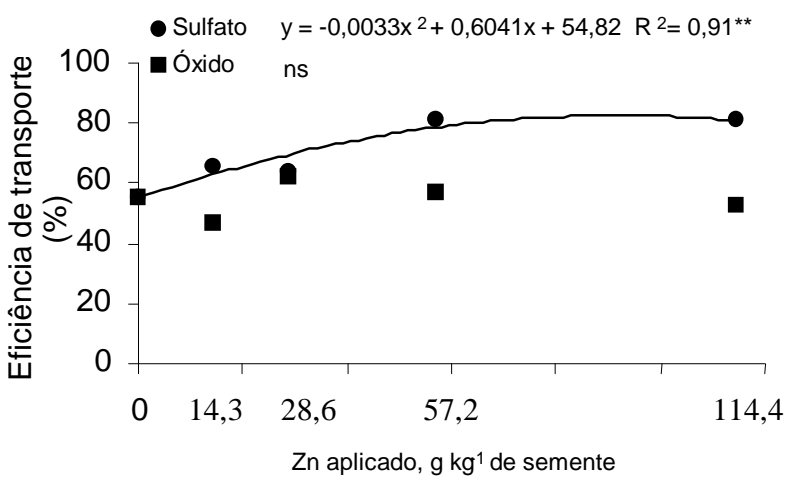

c)

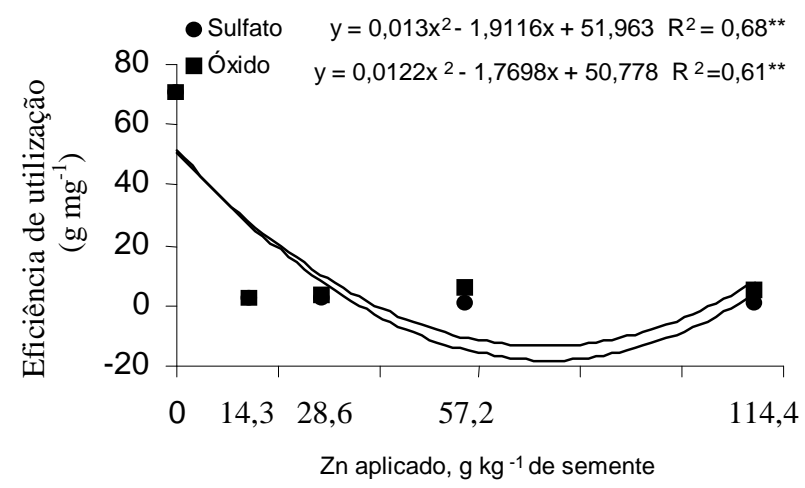

Figura 3. Efeito de fontes de zinco em sementes de sorgo cv. BRS 310 na eficiência de absorção (a), transporte (b) e utilização de zinco (c) aos 25 dias após a semeadura.
Observou-se diferença significativa da eficiência de transporte, em função das doses e das fontes, e da interação (Tabela 2). Porém, os modelos de regressão estudados, não apresentaram ajuste adequado para a fonte óxido (Figura 3b). Em geral, a aplicação de $\mathrm{Zn}$, resultou em aumento significativo da eficiência de transporte do nutriente, apenas com o uso do sulfato, atingindo o máximo de $82 \%$, na dose de $90 \mathrm{~g} \mathrm{~kg}^{-1}$.

O efeito significativo na eficiência de utilização, em função das doses e das fontes de zinco foi verificado, porém não houve interação (Tabela 2). $\mathrm{O}$ aumento das doses de $\mathrm{Zn}$ proporcionou queda na eficiência de utilização, principalmente com uso da fonte sulfato (Figura 3c). A menor queda na eficiência de utilização do $\mathrm{Zn}$ pelo sorgo, com o uso da fonte óxido, ocorreu devido a maior produção de massa seca das plantas, e a menor absorção de Zn, com uso desta fonte. Dessa forma, a aplicação de zinco em sementes, na forma de óxido, resultou em maior eficiência de utilização de $\mathrm{Zn}$, comparada a forma de sulfato (Tabela 2). Provavelmente este efeito do Zn, na forma óxido, contribuiu para maior crescimento inicial do sorgo (Tabela 1).

c) Efeito dos tratamentos sobre o acúmulo dos macronutrientes e micronutrientes ( $\mathrm{B}, \mathrm{Cu}, \mathrm{Fe} e$ $\mathrm{Mn})$

Houve diferença significativa dos fatores doses e fontes de zinco sobre o acúmulo de macro e micronutrientes, na parte aérea do sorgo, exceto o B para fonte (Tabela 3). O maior acúmulo dos macronutrientes, exceto o $\mathrm{S}$ foram observados na ausência de Zn. Este fato deve-se possivelmente a maior produção da massa seca obtida na testemunha (Tabela 3). No que se refere a fonte, observou-se que o sulfato de $\mathrm{Zn}$ proporcionou os maiores acúmulos de $\mathrm{P}, \mathrm{Ca}, \mathrm{S}$ e $\mathrm{Mn}$, enquanto que a fonte de óxido de zinco resultou maior acúmulo de $\mathrm{K}, \mathrm{Mg}, \mathrm{Cu}$ e Fe. 
Tabela 3. Resumo da análise de variância referente ao acúmulo de macro e de micronutrientes na massa seca da parte aérea, em função da aplicação de diferentes fontes e doses de zinco às sementes de sorgo cv. BRS 310, aos 25 dias após a semeadura

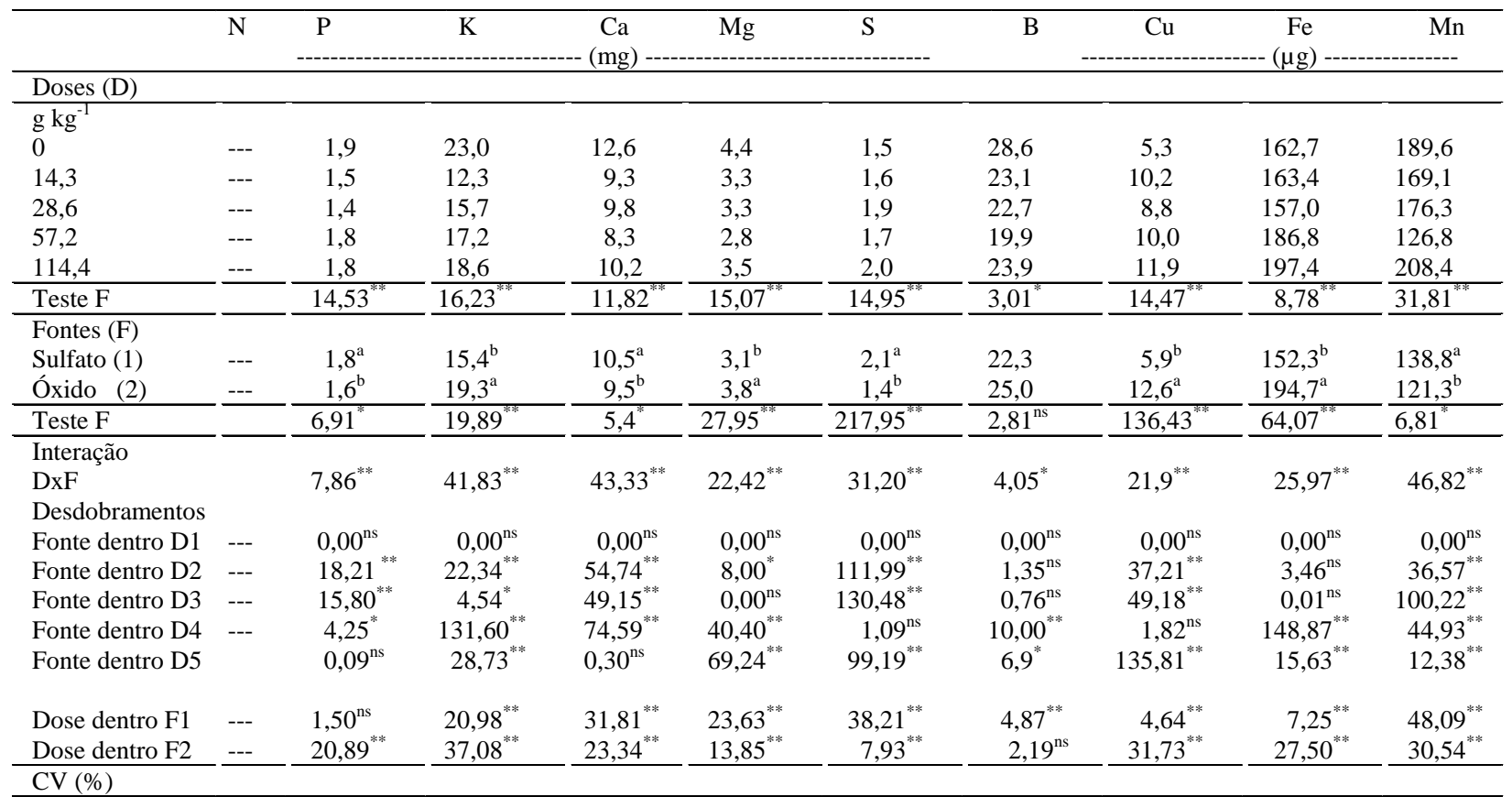

**; *; ns: resultados significativo pelo teste $\mathrm{F}$ a $1 \%$ e $5 \%$ e não significativo a $5 \%$, respectivamente. Letras iguais não diferem entre si pelo teste Tukey a $5 \%$ de probabilidade

Com relação aos resultados de raiz, os tratamentos afetaram significativamente os fatores doses e fonte, para os nutrientes avaliados, exceto para fator dose, o Ca e para fonte o $\mathrm{Mg}$ e Mn. E também houve interação significativa para todos os nutrientes avaliados (Tabela 4). Assim, as doses de zinco, afetaram o acúmulo de $\mathrm{K}, \mathrm{Mg}, \mathrm{Cu}, \mathrm{Fe}$ e $\mathrm{Mn}$ das raízes das plantas (Tabela 4). Observa-se que a maior dose de zinco, proporcionou o maior acúmulo de $\mathrm{Fe}$, sendo que para o Mn, a testemunha apresentou o maior acúmulo do nutriente. E para o fator fontes de zinco, houve efeito significativo para os teores dos nutrientes avaliados, exceto para o $\mathrm{Mg}$ e o $\mathrm{Mn}$.
Observou-se que a aplicação de zinco na forma de óxido elevou a absorção para a maioria dos nutrientes analisados, tanto na parte aérea $(\mathrm{K}, \mathrm{Mg}, \mathrm{Cu}, \mathrm{Fe})$, como na raiz $(\mathrm{K}, \mathrm{Ca}, \mathrm{Cu}, \mathrm{Fe})$. Esse fato, poderia ter contribuído para a maior produção de massa seca do sorgo, com uso da fonte óxido (Tabela 1).

Os estudos de regressão, permitiram observar que aplicação de Zn na forma de sulfato promoveu efeito quadrático no acúmulo dos nutrientes em estudo, exceto o B na parte aérea e $\mathrm{Mg}$ e Fe na raiz que foi linear (Tabela 5). Enquanto, a aplicação de Zn na forma de óxido, promoveu efeito quadrático no acúmulo dos nutrientes da parte aérea e raiz, exceto o $\mathrm{Cu}$ da parte aérea e o $\mathrm{Fe}$ e $\mathrm{Ca}$ da raiz que foi linear (Tabela 5). 
Tabela 4. Resumo da análise de variância referente ao acúmulo de macro e de micronutrientes na massa seca das raízes, em função da aplicação de diferentes fontes e doses de zinco às sementes de sorgo cv. BRS 310, aos 25 dias após a semeadura

\begin{tabular}{|c|c|c|c|c|c|c|c|c|c|c|}
\hline & $\mathrm{N}$ & $\mathrm{P}$ & K & $\mathrm{Ca}$ & $\mathrm{Mg}$ & $\mathrm{S}$ & B & $\mathrm{Cu}$ & $\mathrm{Fe}$ & $\mathrm{Mn}$ \\
\hline \multicolumn{11}{|l|}{ Doses (D) } \\
\hline \multicolumn{11}{|l|}{$\mathrm{g} \mathrm{kg}^{-1}$} \\
\hline 0 & --- & --- & 2,1 & 0,9 & 0,6 & --- & --- & 5,1 & 1398,8 & 70,2 \\
\hline 14,3 & --- & --- & 2,2 & 0,8 & 0,5 & -- & --- & 29,1 & 1278,8 & 41,1 \\
\hline 28,6 & --- & --- & 3,9 & 0,8 & 0,7 & --- & --- & 26,1 & 1353,9 & 56,9 \\
\hline 57,2 & --- & --- & 1,9 & 0,7 & 0,4 & --- & --- & 26,9 & 1484,9 & 39,4 \\
\hline 114,4 & --- & --- & 2,4 & 0,8 & 0,6 & -- & -- & 29,1 & 1904,8 & 57,4 \\
\hline Teste F & & & $21,29^{* * *}$ & $1,50^{\mathrm{ns}}$ & $18,99^{* *}$ & & & $97,88^{* *}$ & $6,68^{\text {*** }}$ & $19,11^{\text {*** }}$ \\
\hline \multicolumn{11}{|l|}{ Fontes $(\mathrm{F})$} \\
\hline Sulfato 1 & --- & --- & $2,3^{\mathrm{b}}$ & $0,7^{\mathrm{b}}$ & 0,5 & --- & --- & $7,5^{\mathrm{b}}$ & $1060,5^{\mathrm{b}}$ & 52,7 \\
\hline Óxido 2 & -- & -- & $2,8^{\mathrm{a}}$ & $1,0^{\mathrm{a}}$ & 0,5 & --- & --- & $41,2^{\mathrm{a}}$ & $1907,5^{\mathrm{a}}$ & 53,3 \\
\hline Teste $\mathrm{F}$ & & & $9,47^{* *}$ & $29,23^{\text {*** }}$ & $3,46^{\mathrm{nS}}$ & & & $1142,15^{* * *}$ & $98,43^{* * *}$ & $0,04^{\mathrm{ns}}$ \\
\hline \multirow{2}{*}{\multicolumn{11}{|c|}{$\begin{array}{l}\text { Interação } \\
\text { DxF }\end{array}$}} \\
\hline & & & $23,25^{* *}$ & $25,09^{* * *}$ & $34,10^{* * *}$ & & & $98,26^{* *}$ & $21,94^{* * *}$ & $49,66^{* *}$ \\
\hline \multicolumn{11}{|l|}{ Desdobramentos } \\
\hline Fonte dentro D1 & --- & --- & $0,00^{\mathrm{ns}}$ & $0,00^{\mathrm{ns}}$ & $0,00^{\mathrm{ns}}$ & --- & --- & $0,00^{\mathrm{ns}}$ & $0,00^{\mathrm{ns}}$ & $0,00^{\mathrm{ns}}$ \\
\hline Fonte dentro D2 & --- & --- & $37,84^{* *}$ & $8,36^{* * *}$ & $13,73^{* *}$ & --- & --- & $556,65^{* * *}$ & $4,78^{*}$ & $31,77^{* * *}$ \\
\hline Fonte dentro D3 & --- & --- & $7,89^{*}$ & $0,30^{\mathrm{ns}}$ & $32,73^{* *}$ & --- & --- & $349,23^{* * *}$ & $1,92^{\mathrm{ns}}$ & $65,46^{* * *}$ \\
\hline Fonte dentro D4 & --- & --- & $43,66^{* * *}$ & $66,89^{* * *}$ & $36,55^{* * *}$ & --- & --- & $110,11^{* *}$ & $56,62^{* * *}$ & $58,25^{* *}$ \\
\hline Fonte dentro D5 & --- & --- & $13,08^{* * *}$ & $54,06^{* * *}$ & $56,87^{* *}$ & --- & --- & $519,59^{* *}$ & $122,89^{* *}$ & $43,22^{* *}$ \\
\hline Dose dentro F1 & --- & --- & $19,27^{* * *}$ & $15,69^{* *}$ & $34,95^{* *}$ & --- & --- & $8,43^{* *}$ & $3,73^{* *}$ & $37,74^{* *}$ \\
\hline Dose dentro F2 & -- & -- & $25,27^{* * *}$ & $10,91^{\text {*** }}$ & $18,14^{* *}$ & -- & -- & $187,81^{* *}$ & $24,89^{* *}$ & $31,03^{* *}$ \\
\hline
\end{tabular}

$\mathrm{CV}(\%)$

**; *; ns: resultados significativo pelo teste $\mathrm{F}$ a $1 \%$ e $5 \%$ e não significativo a $5 \%$, respectivamente. Letras iguais não diferem entre si pelo teste Tukey a $5 \%$ de probabilidade

Tabela 5. Efeito da aplicação de sulfato e óxido de zinco em sementes de sorgo var. BRS 310, sobre o acúmulo de macronutrientes e B, Cu, Fe e Mn na parte aérea e na raiz de plântulas, aos 25 dias após a semeadura.

\begin{tabular}{|c|c|c|c|c|c|c|c|}
\hline \multicolumn{8}{|c|}{ Fonte sulfato } \\
\hline & \multirow[t]{2}{*}{ Nutrientes } & \multicolumn{3}{|c|}{ Parte aérea } & \multicolumn{3}{|l|}{ Raiz } \\
\hline & & Equação & $\mathrm{R}^{2}$ & $\mathrm{~F}$ & Equação & $\mathrm{R}^{2}$ & $\mathrm{~F}$ \\
\hline $\mathrm{N}$ & & ---------------------------------' & ---- & --- & ------------------------- & --- & --- \\
\hline $\mathrm{P}$ & & & & $4,25^{\mathrm{ns}}$ & -------------------------- & --- & --- \\
\hline $\mathrm{K}$ & $\mathrm{g} \mathrm{kg}^{-1}$ & $Y=0,0030 x^{2}-0,439 x+23,78$ & $0,83^{\text {*** }}$ & 84,16 & & & $2,21^{\mathrm{ns}}$ \\
\hline $\mathrm{Ca}$ & & $Y=0,0014 x^{2}-0,211 x+14,51$ & $0,54^{* *}$ & 31,26 & $Y=0,0001 x^{2}-0,017 x+1,05$ & $0,70^{* *}$ & 24,36 \\
\hline $\mathrm{Mg}$ & & $Y=0,0004 x^{2}-0,629 x+4,52$ & $0,96^{* *}$ & 31,97 & $Y=-0,003 x+0,65$ & $0,41^{* *}$ & 14,56 \\
\hline $\mathrm{S}$ & & & & $0,11^{\mathrm{ns}}$ & ---------------------------- & --- & --- \\
\hline B & & $Y=-0,088 x+26,10$ & $0,50^{*}$ & 7,12 & 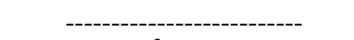 & --- & --- \\
\hline $\mathrm{Cu}$ & $\mathrm{mg} \mathrm{kg}^{-1}$ & $Y=0,0008 x^{2}-0,097 x+4,65$ & $0,40^{* *}$ & 10,01 & $Y=-0,0024 x^{2}+2,66 x+4,65$ & $0,55^{* *}$ & 36,85 \\
\hline $\mathrm{Fe}$ & & $Y=0,013 x^{2}-0,145 x+170,13$ & $0,70^{* *}$ & 53,29 & $Y=-1,44 x+1252,5$ & $0,60^{* *}$ & 6,88 \\
\hline $\mathrm{Mn}$ & & $Y=0,0176 x^{2}-2,37 x+220,58$ & $0,30^{* *}$ & 64,73 & $Y=0,0061 x^{2}-1,10 x+77,27$ & $0,50^{* *}$ & 20,78 \\
\hline \multicolumn{8}{|c|}{ Fonte óxido } \\
\hline $\mathrm{N}$ & & 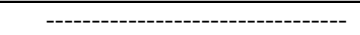 & -- & -- & |----------------------- & --- & --- \\
\hline $\mathrm{P}$ & & & & $4,35^{\mathrm{ns}}$ & --------------------------- & --- & --- \\
\hline $\mathrm{K}$ & $\mathrm{g} \mathrm{kg}^{-1}$ & & & $0,1^{\mathrm{ns}}$ & $Y=-0,0004 x^{2}+0,054 x+1,75$ & $0,30^{* *}$ & 15,11 \\
\hline $\mathrm{Ca}$ & & & & $4,03^{\mathrm{ns}}$ & $Y=0,005 x+0,76$ & $0,65^{* *}$ & 17,94 \\
\hline $\mathrm{Mg}$ & & $Y=0,0003 x^{2}-0,024 x+3,89$ & $0,57^{* *}$ & 13,82 & & & $0,08^{\mathrm{ns}}$ \\
\hline $\mathrm{S}$ & & & & $0,07^{\mathrm{ns}}$ & ---------------- & --- & --- \\
\hline B & & & & $2,65^{\mathrm{ns}}$ & ------------------------- & -- & --- \\
\hline $\mathrm{Cu}$ & $\mathrm{mg} \mathrm{kg}^{-1}$ & $Y=0,088 x+8,82$ & $0,60^{* *}$ & 59,85 & $Y=-0,0055 x^{2}+0,820 x+23,96$ & $0,30^{* *}$ & 24,58 \\
\hline $\mathrm{Fe}$ & & $Y=-0,0126 x^{2}+2,14 x+146,94$ & $0,61^{* *}$ & 11,76 & $Y=14,62 x+1280,1$ & $0,96^{* *}$ & 61,65 \\
\hline $\mathrm{Mn}$ & & $Y=0,0184 x^{2}-1,58 x+171,8$ & $0,61^{* *}$ & 38,09 & $Y=0,0065 x^{2}-0,502 x+52,2$ & $0,61^{* *}$ & 18,58 \\
\hline
\end{tabular}

**;* e ns - Significativo a $1 \%$ e a $5 \%$ de probabilidade, e não significativo, respectivamente. 


\section{Conclusões}

A aplicação de zinco na forma de óxido promoveu maior incremento de massa seca em plantas de sorgo e proporcionou maior eficiência de utilização do micronutriente, embora, com menor eficiência de absorção, quando comparado a fonte sulfato.

A fonte sulfato causou diminuição na produção da massa seca do sorgo.

As fontes promoveram alteração na absorção de outros nutrientes pelas plantas de sorgo, sendo que a fonte sulfato incrementou a absorção de $\mathrm{P}, \mathrm{Ca}, \mathrm{S}$ e Mn e a fonte óxido maior absorção de $\mathrm{K}, \mathrm{Mg}, \mathrm{Cu}$ e $\mathrm{Fe}$.

\section{Agradecimentos}

À FAPESP pelo auxílio à Pesquisa concedido (Processo 2004/14662-6).

\section{Referências}

ALVAREZ V. V.H.; DEFELIPO, B. V.; BARROS, N. F. Resposta do sorgo à aplicação de micronutrientes num Latossolo Vermelho-Amarelo de Itamarandiba, Minas Gerais. Ceres, São Paulo, v.25, p.79-86, 1978.

BATAGLIA, O. C.; FURLANI, A. M. C.; TEIXEIRA, J. P. F.; FURLANI, P. R.; GALLO, J. R.. Métodos de análise química de plantas. Campinas: Instituto Agronômico, 1983. (Boletim Técnico, 78).

CANTARELLA, H.; RAIJ, B. V.; SAWAZAKI, E. Sorgogranífero, forrageiro e vassoura. In: RAIJ, B.V.; CANTARELLA, H.; QUAGGIO, J.A.; FURLANI, A.M.C. (Eds.). Recomendações de adubação e calagem para o estado de São Paulo. 2.ed. Campinas: Instituto Agronômico, 1997. p.66-67 (Boletim Técnico, 100).

HOAGLAND, D. R.; ARNON, D. I. The water culture method for growing plants without soil. Berkeley: The College of Agriculture, University of California, [1950]. (California. Agricultural Experiment Station. Circular, 347).
LOCKMAN, R. B. Mineral composition of grain sorghum plant samples. Part II: As affected by soil acidity, soil fertilitiy, stage of growth, variety, and climate fators. Communications in Soil Science and Plant Analysis, New York, v.3, p.283-293, 1972.

LOPES, A. S. Solos sob cerrado: características, propriedades e manejo. Piracicaba: Potafos, 1983.

MALAVOLTA, E.; VITTI, G. C.; OLIVEIRA, S. A. Avaliação do estado nutricional das plantas: princípios e aplicações. Piracicaba: POTAFOS, 1997.

MARSCHNER, H. Mineral nutrition of higher plants. 2nd San Diego: Academic, 1995.

MENGEL, K.; KIRKBY, E. A. Principles of plant nutrition. Bern: International Potash Institute, 1987. p.525-536.

RIBEIRO, N. D.; SANTOS, O. S. Aproveitamento do zinco aplicado na semente na nutrição da planta. Ciência Rural, Santa Maria, v.26, p.159-165, 1996.

RIBEIRO, N. D.; SANTOS, O. S.; MENEZES, N. L. Efeito do tratamento com fontes de zinco e boro na germinação e vigor de sementes de milho. Scientia Agricola, Piracicaba, v.51, p.481-485, 1994.

RITCHEY, K. D.; COX, F. R.; GALRÃO, E.Z.; YOST, R. S. Disponibilidade de zinco para as culturas do milho, sorgo e soja em Latossolo Vermelho-Escuro argiloso. Pesquisa Agropecuária Brasileira, Brasília, v.21. p.215-225, 1986.

SAS INSTITUTE. SAS/STAT procedure guide for personal computers. 5.ed. Cary: Sas Institute, 1996.

SIDDIQI, M. Y.; GLASS, A. D. M. Utilization index: a modified approach to the estimation and comparison of nutrient utilization efficiency in plants. Journal of Plant Nurition, New York, v.4, p.289-302, 1981.

VALE, F. Avaliação e caracterização da disponibilidade do boro e zinco contidos em fertilizantes. 2001. Tese (Doutorado) - Escola Superior de Agricultura "Luiz de Queiroz" - Universidade de São Paulo, Piracicaba.

YAGI, R. M.; SIMILI, F. F.; ARAÚJO, J. C.; PRADO, R. M.; SANCHEZ, S. V.; RIBEIRO, C. E. R.; BARRETO, V. C. M. Aplicação de zinco via sementes e seu efeito na germinação, nutrição e desenvolvimento inicial do sorgo. Pesquisa Agropecuária Brasileira, Brasília, v.41, n.4, p.655-660, 2006. 